\title{
Medical cases who would benefit from treatment on a spinal injury unit
}

Spinal Cord (2004) 42, 435-437. doi:10.1038/sj.sc.3101582

At the beginning of the Second World War, specialised units were established to treat servicemen with traumatic spinal injuries in the UK but, from the outset, patients with nontraumatic injuries were admitted and have always constituted an important element of the work.

At Stoke Mandeville, founded in 1944, of the first 2000 patients, 1349 were traumatic admissions and 651 were nontraumatic. ${ }^{1}$ This work has continued and in 2000 in the UK as a whole, total admissions were 825 of which 666 were traumatic and 159 were nontraumatic. $^{2}$

Misconceptions grew up which have persisted: spinal units only deal with traumatic cases and are too busy to admit nontraumatic cases; they are rehabilitation units with little to offer to patients with other neurological disabilities; the regime of treatment is too physically demanding.

What is the approach on a spinal unit? There is a holistic approach. Problems are addressed by a multidisciplinary team. A severe pressure sore in a spastic lower limb cannot be dealt with until the spasticity is reduced because constant movement will prevent healing and this may require the combined approach of an orthopaedic surgeon, neurosurgeon and a plastic surgeon. Pressure sores are dealt with very well on spinal units. While plastic surgery is available on plastic units, the specialised needs of the paralysed patient are not always appreciated.

Specialised training skills are available to deal with bladder problems, with a designated urologist, backed up by urodynamic facilities, with time allotted in the $\mathrm{X}$-ray department and operating theatres. Specific operations are available to deal with incontinence such as stents and augmentation cystoplasty. The congregation of large numbers of patients with neuropathic bladders enables skills and treatment to be developed for traumatic spinal cases, which can then be applied to the nontraumatic cases

The specialised nursing skills both from a technical and psychological viewpoint are keystone.

Specialised wheelchair and walking skills are available. Physiotherapists on spinal units are more experienced than those in the community and have higher ambitions for their patients and better facilities. There is a seating clinic with pressure studies to see if patients have the right cushion, and a gait clinic.

On a spinal unit, adequate time is set aside for a structured programme. Patients are encouraged because they can see they are progressing day by day and can compare themselves with other patients with a similar condition.

The occupational therapists have particular skills in helping patients reach their maximum level of function and independence in all aspects of daily life, for example by organising equipment to surmount feeding difficulties, suggesting modifications to the home and assessing driving.

The clinical psychologist can assess mental impairment, dealing with patients' lack of motivation and reconciling them to the problems that have to be surmounted.

Social workers with particular skills in dealing with paralysed patients can also direct patients to appropriate teams for counselling and advice on sexual problems. These particular needs of sexual dysfunction are not recognised and seldom addressed outside specialised spinal units.

The treatment of spasticity is difficult and time consuming. The spinal unit, where the patient can be admitted and remain under surveillance, is ideal for assessing the relative role of physiotherapy and drug treatment, both orally and intrathecally. The assessment can be carried out by a combination of physiotherapists and nurses. If an operation is to be performed, the postoperative management and splinting can be supervised by the same team.

All patients need targets and to be told what to do but such goal setting requires time and space with a multidisciplinary team approach, which is seldom available in the community.

There is a danger that patients, while undergoing rehabilitation, will become institutionalised and isolated from their family. The ideal pattern is to have smaller units enabling patients to be rehabilitated close to their homes so that they can spend weekends at home and this can be supervised by a peripatetic nurse.

Preferably, patients will be assessed by a spinal injury consultant in conjunction with the referring consultant. Subsequent problems can be dealt with on a combined round with the appropriate specialist and case conferences held with the whole team, the General Practitioner and the patient's family.

While rehabilitation is available outside spinal units, it is fragmented. In the community, the patient is isolated. There are no role models in the community. One cauda equina patient when shown how to use a 
catheter and regulate her bowels thought she was the only person in the world that had to do this.

There is only one neurologist in the UK to deal with every 160000 patients. Consequently, physicians may not have the knowledge or the time to implement an effective programme of rehabilitation. Rehabilitation of young adults with neurological disability in the UK is restricted. In the UK, about 100000 patients have a first stroke every year and about a quarter of these occur below the age of 65 years. $^{3}$ Even acute referrals with the first episode of multiple sclerosis may have to wait 6 months to be seen so there is little time available to supervise chronic rehabilitation. Physiotherapists in the community have little support from neurologists.

Patients with multiple sclerosis and spina bifida do not get the attention they deserve. They may be seen by a urologist for their bladder problems or be seen by a physiotherapist but they are seldom treated comprehensively. The wait between consultations with different specialists can be so long that by the time they are seen the predominant problem may have changed and the patient becomes demoralised. Patients may be referred to a urologist because of incontinence but by the time they are seen they may have developed pressure sores and very severe spasms.

Patients with nonprogressive lesions of the cord such as transverse myelitis, spinal artery thrombosis or benign tumours are ideal candidates for a spinal unit.

In the case of secondary deposits causing paraplegia, previously unrecognised widespread secondaries in the lungs or brain may overtake the patient before rehabilitation can be achieved. Even when it does not, patients may be in pain, debilitated and unable to participate in the programme. This makes these patients very unhappy because they cannot achieve as much as the patients with traumatic injuries and this will have a demoralising effect on the staff and other patients. If a discreet short programme can be established concentrating on obtaining a suitable wheelchair and cushion, mobilising the patient and getting them home, this will be very rewarding.

Age is not a contradiction to rehabilitating patients with nonprogressive spinal cord injuries but intercurrent illnesses may overtake them.

Patients with spina bifida have particular problems. Initially, they are dealt with by the paediatricians but in adult life they may be lost to follow-up or pass to the care of neurologists or neurosurgeons who do not have sufficient time to undertake comprehensive care.

In severe cases their lower limbs have never formed properly and they are often small with heavy, obese bodies. Since they have never walked on their limbs they regard them differently to paraplegics.

There are particular problems with their bladder and bowels that require the specialised continued care of a urologist and colorectal surgeon. As a result of their deformity, they need specialised seating and attention from a team including a plastic surgeon to deal with the pressure sores.
They may be in hospital for many years having a series of operations and become very dependent, or, they may be discharged home to the care of their parents who mollycoddle them. There may be resistance on the part of both the patient and their relatives to make them independent, so there are psychological problems that need to be overcome.

If patients with multiple sclerosis have mental impairment, they will be unable to understand instructions and will not benefit from treatment. If there is a particular problem, such as ataxia, they will fatigue easily but, provided their condition is not rapidly progressive, they will benefit from attending a spinal unit.

In this day of audit, clinical governance and evidence based medicine, treatments are being examined in a very critical manner with double blind control trials and have to be shown to be therapeutically and financially advantageous.

Is there any evidence, apart from anecdotes, that treatment on a spinal injury unit is beneficial $?^{4-10}$ It has been shown that early referral of a patient with a traumatic spinal injury lessens the complications, shortens the length of time in hospital and is therefore cheaper. Evidence is not available for medical cases in matched controls. A matched series of medical cases admitted early to a spinal unit compared with another group cannot be produced for ethical and logistic reasons. Kesselring and Beer ${ }^{11}$ reviewed the evidence in cases of multiple sclerosis and concluded that there were no studies to fulfil the criteria of evidence-based medicine, but there was good clinical evidence for the efficacy of rehabilitation on an inpatient basis.

This picture of treatment refers to a developed country such as the UK. It does not refer to underdeveloped countries where there are no spinal units or to Continental Europe where the rehabilitation service is well established and the coordination of various specialities needed for comprehensive management works at regional or national in addition to institutional level and there is not the need for independent institutions of comprehensive units. The arrangements in the US are complex, there being a mixture of excellent free treatment in the Veterans Hospitals and certain limited facilities on a county basis. Other than that, treatment is covered by private insurance. Consequently not all patients with spinal cord disorders receive comprehensive care.

Unfortunately spinal units in the UK, like the rest of the Health Service, have the greatest difficulty in admitting patients because of MRSA infection, shortage of funds, blockage of beds and shortage of staff. In 2001, Stoke Mandeville had a waiting list of 47 patients (report in The Bucks Herald, 23 October 2002) and this has now increased and is a universal problem. Even in Sheffield, where early admission was pioneered, there is now a waiting list of 17 .

This dire situation cannot persist indefinitely and should not deter practitioners from referring patients 
with medical causes of paralysis to spinal units where they unquestionably benefit from the treatment.

\section{Acknowledgements}

I thank the following for their help in preparing this article. Dr D Badwan, Ebba Bergstrom, Sister Diane Cooper, Dr A Graham, Mr David Grundy, Dr Paul Kennedy, Mr G Ravichandran, Lone Rose and Dr D Short

JR Silver

National Spinal Injuries Unit, Stoke Mandeville Hospital, Aylesbury, Bucks, UK

\section{References}

1 Guttmann L. Spinal Cord Injuries Comprehensive Management and Research. Blackwell: London: 1973, pp 627-628.

2 Spinal Injuries Association. Annual Report 2001-2002.
3 Donaghy M (ed). Brain's Diseases of the Nervous System, 11 th edn. Oxford University Press, Oxford, p 779.

4 Damanski M. Heterotopic ossification in paraplegia. J Bone Joint Surg 1961; 43B: 286-299.

5 Silver JR. Heterotopic ossification. A clinical study of its possible relationship to trauma. Paraplegia 1969; 7: 220-230.

6 Thiyagarajan C, Silver JR. Aetiology of pressure sores in patients with spinal cord injury. $B M J$ 1984; 289: 1487-1490.

7 Stover SL et al. Spinal Cord Injury: The Facts and Figures. The University of Alabama: Birmingham, Alabama: 1986, p 44.

8 Daud O, Sett P, Burr RG, Silver JR. The relationship of heterotopic ossification to passive movements in paraplegic patients. Disab Rehabil 1993; 15: 114-118.

9 Aung TS, El Masry WS. Audit of a British Centre for spinal injury. Spinal Cord 1997; 35: 147-150.

10 Smith M. Efficacy of specialist versus non-specialist management of spinal cord injury within the U.K. Spinal Cord 2002; 40: 11-16.

11 Kesselring J, Beer S. Rehabilitation in Multiple Sclerosis. ACNR 2002; 2: 6-8. 